Original

\title{
Validez de los índices de masa corporal y de masa grasa como indicadores de sobrepeso en adolescentes españoles: estudio Esccola
}

\author{
José Ramón Alvero-Cruz ${ }^{\mathrm{a}, *}$, Elvis Álvarez Carnero ${ }^{\mathrm{a}, \mathrm{b}}$, José Carlos Fernández-García ${ }^{\mathrm{a}}$, Jesús Barrera \\ Expósito ${ }^{\mathrm{a}}$, Margarita Carrillo de Albornoz Gil ${ }^{\mathrm{a}}$ y Luis B. Sardinha ${ }^{\mathrm{b}}$
}

${ }^{a}$ Escuela de Medicina de la Educación Física y del Deporte, Facultad de Medicina, Universidad de Málaga, Málaga, España

${ }^{\mathrm{b}}$ Health and Exercise Laboratory, Faculty of Human Movement, Technical University of Lisbon, Lisboa, Portugal

\section{INFORMACIÓN DEL ARTÍCULO}

\section{Historia del artículo:}

Recibido el 24 de julio de 2009

Aceptado el 5 de enero de 2010

On-line el 26 de marzo de 2010

\section{Palabras clave:}

Índice de masa corporal

Índice de masa grasa

Índice de masa libre de grasa

Curvas receiver operating characteristics

Sensibilidad

Especificidad

\begin{abstract}
R E S U M E N
Objetivo y fundamento: Comprobar la exactitud del índice de masa corporal (IMC), del índice de masa grasa (IMG) y del índice de masa libre de grasa (MLG) para diagnosticar y determinar valores de corte para el sobrepeso en una población de jóvenes adolescentes entre 12 y 18 años de edad.

Sujetos y método: Se estudió a 150 niños (75 varones: edad media de 14,81 años [desviación estándar \{DE\} de 1,86 ], talla media de $168,8 \mathrm{~cm}$ [DE de 8,8 ], peso medio de $65,84 \mathrm{~kg}$ [DE de 16] e IMC medio de $22,89 \mathrm{~kg} / \mathrm{m}^{2}$ [DE de 4,4]; 75 niñas: edad media de 14,88 años [DE de 1,94], talla media de 159,4 cm [DE de 7,6], peso medio de $54,77 \mathrm{~kg}$ [DE de 9,54] e IMC medio de $21,48 \mathrm{~kg} / \mathrm{m}^{2}$ [DE de 3,1]). El porcentaje de masa grasa se determinó mediante el cálculo del agua corporal total con un espectrómetro de masas para razones estables y aplicando las constantes de hidratación de la MLG para cada edad. La definición de sobrepeso se realizó aplicando los valores de porcentaje de grasa de Taylor relativos a cada edad y correspondientes al punto de corte internacional de $25 \mathrm{~kg} / \mathrm{m}^{2}$, que clasifica el estado de sobrepeso. Se aplicó un análisis de curvas receiver operating characteristics y se calcularon las áreas bajo la curva de cada índice (IMC, IMG y MLG), así como los valores de sensibilidad y especificidad.

Resultados: En los niños, los valores predictivos positivos fueron del 78,1\% para el IMC y del 89,2\% para el IMG, mientras que para las niñas estos valores fueron del 34,8 y del $81,4 \%$, respectivamente. Los valores predictivos negativos fueron del 81,4 y del $100 \%$ en los niños y del 98,1 y del $100 \%$ en las niñas para el IMC y el IMG, respectivamente. Las áreas bajo la curva del IMG para los niños $(0,988$, intervalo de confianza del 95\% de $0,930-0,998)$ y las niñas $(0,996$, intervalo de confianza del $95 \%$ de $0,943-1,000)$ mostraron una alta capacidad de predicción para el diagnóstico de sobrepeso. Los puntos de corte del IMG en los niños de $4,58 \mathrm{~kg} / \mathrm{m}^{2}$ y en las niñas de $7,76 \mathrm{~kg} / \mathrm{m}^{2}$, y los del IMC de $23,07 \mathrm{~kg} / \mathrm{m}^{2}$ en los niños y de $23,18 \mathrm{~kg} / \mathrm{m}^{2}$ en las niñas poseyeron una gran sensibilidad y especificidad como indicadores de sobrepeso en adolescentes.

Conclusiones: Los resultados muestran que el IMG tiene una precisión muy alta para diagnosticar el estado de sobrepeso y que los puntos de corte se presentan como una herramienta válida en estudios clínicos y epidemiológicos para la detección de sobrepeso en la población adolescente.

๔ 2009 Elsevier España, S.L. Todos los derechos reservados.
\end{abstract}

\section{Validity of body mass index and fat mass index as indicators of overweight status in Spanish adolescents: Esccola Study}

\section{A B S T R A C T}

Background and objectives: To determine the accuracy of body mass index (BMI), fat mass index (FMI) and fat-free mass index (FFMI) to find cut-off points related to overweight in adolescents aged 12-18 years. Subjects and method: 150 adolescents were assessed $(75$ boys aged $14.81+1.86 \mathrm{yr}$, height $168.8+8.8 \mathrm{~cm}$ weight $65.84 \pm 16 \mathrm{~kg}$ and BMI $22.89 \pm 4.4 \mathrm{~kg} \cdot \mathrm{m}^{2}$, and 75 girls aged $14.88 \pm 1.94 \mathrm{yr}$, height $159.4 \pm 7.6 \mathrm{~cm}$, weight $54.77 \pm 9.54 \mathrm{~kg}$ and BMI $21.48 \pm 3.1 \mathrm{~kg} \cdot \mathrm{m}^{2}$ ). Body composition was measured by using the deuterium dilution technique with an isotope ratio mass spectrometer and fat free mass hydration constants were applied.

Taylor's age-related overweight classification was applied and receiver operating characteristic (ROC) curves analyses were performed. Areas under curve were calculated for every index (BMI, FMI and FFMI). Sensitivity and specificity values of BMI and FMI to diagnose overweight were evaluated.

Results: Among boys, predictive positive values for BMI were $78.1 \%$ and $89.2 \%$ for FMI; predictive positive values in girls was $34.8 \%$ for BMI and $81.4 \%$ for FMI. Predictive negative values were $81.4 \%$ and $100 \%$ in boys and $98.1 \%$ and $100 \%$ in girls for BMI and FMI. Areas under curve of FMI for boys

\footnotetext{
* Autor para correspondencia.

Correo electrónico: alvero@uma.es (J.R. Alvero-Cruz).
} 
(0.988, 95\%CI: $0.930-0.998)$ and girls (0.996, 95\%CI: $0.943-1.000)$ showed high accuracy for overweight diagnose. Cutoff points of $4.58 \mathrm{~kg} . \mathrm{m}^{2}$ in boys and $7.76 \mathrm{~kg} . \mathrm{m}^{2}$ in girls for FMI and $23.07 \mathrm{~kg} . \mathrm{m}^{2}$ in boys and $23.18 \mathrm{~kg} . \mathrm{m}^{2}$ in girls for BMI had high sensitivity and specificity as indicators of overweight classification. Conclusions: FMI showed high accuracy for overweight screening. Thus, our cut-off points may be useful as a tool to surveillance children's overweight on clinical and epidemiologic settings.

(c) 2009 Elsevier España, S.L. All rights reserved.

El sobrepeso y la obesidad representan un cuadro clínico patológico crónico caracterizado por un aumento de la masa grasa corporal, lo que se ha asociado al desarrollo de otras comorbilidades de forma independiente, como la hipertensión arterial, la dislipidemia, la diabetes mellitus tipo II, la cardiopatía isquémica y algunos tipos de cáncer ${ }^{1,2}$. Por esto, el control del incremento de la adiposidad representa un parámetro más de la evaluación clínica habitual, por su fuerte asociación a numerosos riesgos de salud. No obstante, la evaluación de la cantidad de masa grasa representa aún hoy una tarea difícil fuera de los laboratorios, y por esto se han adoptado otras formas simples y fiables de estimarla, bien para un diagnóstico inicial o bien para el control longitudinal.

La Organización Mundial de la Salud ${ }^{1}$ ha propuesto el índice de masa corporal (IMC) de Quetelet (calculado como el peso en kilogramos dividido por el cuadrado de la estatura en metros) como el parámetro para el diagnóstico de sobrepeso y obesidad, en el que considera su buena asociación a la adiposidad total y su fuerte correlación epidemiológica con la morbimortalidad asociada a la obesidad ${ }^{3}$. Sin embargo, la exactitud del IMC para estimar la composición corporal es discutible ${ }^{4}$ debido a que está influida por el sexo, la raza y la edad, lo que puede conducir a una clasificación errónea del estado de sobrepeso u obesidad ${ }^{5}$.

Es así que individuos diagnosticados como normales según el IMC pueden tener un porcentaje de masa grasa (\%MG) elevado (falsos negativos), mientras que otros, diagnosticados como obesos por un IMC mayor a $30 \mathrm{~kg} / \mathrm{m}^{2}$, pueden tener un porcentaje de grasa dentro de límites de normalidad (falsos positivos). En el caso de los niños y los jóvenes, la aparición de falsos negativos es común debido a que el crecimiento en longitud y superficie se dan de forma natural debido al crecimiento y no sólo como resultado del desequilibrio energético. Por esto, los valores utilizados para adultos no representan una forma fiable y válida para niños y adolescentes en proceso de crecimiento y maduración. No obstante, la dificultad para estimar la masa grasa en jóvenes, debido a la complejidad de las técnicas, requiere un alto grado de colaboración del sujeto. De esta forma, la mayor parte de los estudios continúa utilizando el IMC como índice de grasa corporal $^{6}$ y se lo ha recomendado como el mejor índice para definir la obesidad en jóvenes adolescentes ${ }^{7}$ por su relativa facilidad y precisión frente a otras medidas como pliegues de grasa y perímetros, que requieren mayor capacidad y entrenamiento. Sin embargo, a pesar de existir nuevos valores de IMC de niños y adolescentes, éste continúa siendo un estimador indirecto de la adiposidad y existen numerosas limitaciones que pueden llevar a interpretaciones erróneas, especialmente en niños en proceso de maduración. Así, la masa corporal total se compone de la masa grasa y la masa libre de grasa $(M L G)^{8}$ y ambos componentes puede tener diferencias entre individuos debido a la variabilidad de componentes de la MLG (proteínas, agua y minerales) en niños y adolescentes. El \%MG está influido por la cantidad total de MLG y el IMC no es un índice independiente de la masa corporal.

El sobrepeso y la obesidad producen un incremento adicional de las MLG y de la propia masa grasa. Así, la expresión de la grasa corporal en el niño obeso y el porcentaje de grasa estimarán la baja la cantidad absoluta de la masa grasa ganada y ocultarán la variabilidad en la MLG. Para intentar resolver esta cuestión, tanto la masa grasa como la MLG se pueden normalizar por la estatura mediante el IMC y el índice de masa grasa (IMG) ${ }^{9}$.
Se parte del IMC como IMC en relación con la talla. El IMC se puede descomponer en la siguiente fórmula: $\operatorname{IMC}\left(\mathrm{kg} / \mathrm{m}^{2}\right)=$ peso $(\mathrm{kg}) /$ talla $(\mathrm{m})^{2}=$ MLG $(\mathrm{kg}) /$ talla $(\mathrm{m})^{2}+$ masa grasa $(\mathrm{kg}) /$ talla $(\mathrm{m})^{2}$. Estos 2 índices, conocidos como índice de MLG (IMLG) e IMG, están ajustados a la talla del individuo ${ }^{9,10}$, y tienen una gran especificidad y una gran sensibilidad en el diagnóstico y el control del cambio ponderal en niños ${ }^{10}$.

El objetivo principal de este estudio fue evaluar el grado de sensibilidad y especificidad del IMC, del IMG y del IMLG como herramienta para diagnosticar correctamente el sobrepeso en adolescentes de $12-18$ años de edad de ambos sexos.

\section{Sujetos y método}

\section{Sujetos}

La población de estudio estuvo formada por 150 jóvenes (75 niños y 75 niñas) de $12-18$ años de edad. Se eligió el centro escolar IES Nuestra Sra. de la Victoria, de la ciudad de Málaga, que se consideró representativo de la población escolarizada de esas edades (por su distribución según el nivel socioeconómico y las características demográficas). Dentro del centro escolar, los sujetos se seleccionaron por muestreo aleatorizado simple para el intervalo de edad de $12-18$ años. La selección se realizó durante el período de octubre a diciembre de 2006. Las características físicas de los sujetos se resumen en la tabla 1. A todos los sujetos se les realizó una serie de exploraciones médicas y físicas y diversas encuestas para caracterizar sus hábitos alimentarios y de actividad física ${ }^{11}$; todos los sujetos se encontraban exentos de enfermedades agudas o crónicas y, en el momento de las exploraciones, no tomaban medicación alguna. Se explicó extensamente la naturaleza y el objetivo de estas exploraciones a los profesores, al Consejo Escolar y a las asociaciones de madres y padres de alumnos. El Comité de Ética de la Facultad de Medicina de la Universidad de Málaga aprobó todos los procedimientos. Antes del estudio, los responsables de la educación de los sujetos procedieron a la firma de los consentimientos informados, en los que se explicaron

Tabla 1

Características generales y composición corporal de la población estudiada*

\begin{tabular}{lccl}
\hline Variable & Niños $(\mathbf{n = 7 5})$ & Niñas $(\mathbf{n = 7 5 )}$ & $\mathbf{p}$ \\
\hline Edad, años & $14,81 \pm 1,86$ & $14,88 \pm 1,94$ & $\mathrm{~ns}$ \\
Estatura, cm & $168,80 \pm 8,80$ & $159,40 \pm 7,60$ & 0,001 \\
Peso, kg & $65,84 \pm 16,04$ & $54,77 \pm 9,54$ & 0,001 \\
Sumatorio de 4 pliegues, mm & $58,97 \pm 30,08$ & $63,86 \pm 20,97$ & $\mathrm{~ns}$ \\
Sumatorio de 6 pliegues, mm & $92,52 \pm 46,75$ & $108,51 \pm 32,47$ & 0,01 \\
Sumatorio de 8 pliegues, mm & $119,09 \pm 59,56$ & $136,12 \pm 42,26$ & 0,045 \\
MG, kg & $14,21 \pm 8,82$ & $15,18 \pm 5,21$ & $\mathrm{~ns}$ \\
MLG, kg & $51,64 \pm 9,76$ & $39,60 \pm 6,32$ & 0,001 \\
IMC, kg/m ${ }^{2}$ & $22,90 \pm 4,43$ & $21,49 \pm 3,12$ & 0,026 \\
\%MG & $20,37 \pm 8,10$ & $27,24 \pm 6,04$ & 0,001 \\
IMG, kg & $4,94 \pm 2,91$ & $5,94 \pm 1,89$ & 0,001 \\
IMLG, kg/m ${ }^{2}$ & $17,96 \pm 2,23$ & $15,55 \pm 2,11$ & 0,001 \\
\hline
\end{tabular}

\%MG: porcentaje de masa grasa; IMC: índice de masa corporal; IMG: índice de masa grasa; IMLG: índice de masa libre de grasa; MG: masa grasa; MLG: masa libre de grasa; ns: no significativo.

* Valores expresados como media \pm desviación estándar. 
detalladamente todos los instrumentos y metodologías que se utilizarían durante la recogida de datos.

\section{Evaluación antropométrica y de composición corporal}

Se midió el peso (en $\mathrm{kg}$ ) utilizando una balanza digital Seca 770 (Hamburg, Alemania), con una precisión de $0,1 \mathrm{~kg}$, y la estatura (en $\mathrm{cm}$ ) mediante un tallímetro de pared Seca 208 (Hamburg, Alemania), con una precisión de $1 \mathrm{~mm}$. Para el cálculo del IMC se dividió el peso en kilogramos por la talla en metros al cuadrado $\left(\mathrm{IMC}=\mathrm{kg} / \mathrm{m}^{2}\right)$. Para el cálculo del IMLG se dividió el peso libre de grasa en kilogramos por la talla en metros al cuadrado $\left(\mathrm{IMLG}=\mathrm{kgMLG} / \mathrm{m}^{2}\right)$. Para el cálculo del IMG se dividió el peso en kilogramos por la talla en metros al cuadrado $\left(\mathrm{IMG}=\mathrm{kgMG} / \mathrm{m}^{2}\right)$. Se procedió a la toma por duplicado de las variables antropométricas según criterios internacionales, estandarizados por la International Society for Advancement in Kinanthropometry ${ }^{12}$.

\section{Estimación de la masa grasa}

El cálculo del \%MG se realizó a través de la estimación de la MLG por hidrometría. El principio de la hidrometría postula que la MLG guarda una relación de proporcionalidad constante con el agua corporal total (ACT) para sujetos con la misma maduración biológica. De esta forma, al conocer esta última podemos derivar la primera. Así, la estimación de la MLG se realizó utilizando un modelo de 2 componentes $(\mathrm{MLG}=\mathrm{ACT} /$ constantes de hidratación de la MLG), donde las constantes de hidratación de la MLG se aplicaron cada medio año de edad según las propuestas de Fomon et $\mathrm{al}^{13}$ y Lohman ${ }^{14}$, ya que la maduración bioquímica de los niños y adolescentes difiere de la de los adultos, y en este caso, la hidratación de la MLG disminuye de forma significativa con el avance de la edad. El cálculo final del \%MG se realizó de forma tradicional $(\% \mathrm{MG}=[$ peso corporal-MLG]/peso corporal $\times 100)$. De acuerdo con los párrafos previos, la única variable de interés que necesitamos medir es el ACT, que se realizó aplicando el método de dilución utilizando una solución acuosa de óxido de deuterio (D2O) al 99,9\% (Aldrich, Alemania) para marcar el agua corporal, que se considera uno de los métodos de referencia para la evaluación de la composición corporal in vivo ${ }^{15}$. A continuación se describe brevemente su procedimiento. Los sujetos llegaron a nuestras instalaciones a primera hora de la mañana en ayunas, sin haber ingerido agua ni ningún otro líquido esa mañana; después de realizar una primera medición del peso corporal, se recogió una primera muestra de orina en un frasco esterilizado. Se preparó una solución individual de agua y deuterio para cada sujeto con el fin de administrar una dosis única por vía oral de $0,1 \mathrm{~g}$ de D2O.kg-1 de peso corporal (estas dosis se han utilizado ampliamente en la literatura médica y se las considera válidas y seguras para estas edades; en este estudio, ningún sujeto manifestó malestar o cualquier efecto secundario asociado a la ingesta de esa solución durante la administración o en los días siguientes a la administración). Después de la toma, discurrió un período de equilibrio y dilución en los fluidos corporales de $4 \mathrm{~h}$, donde se mantuvo a los sujetos en reposo y realizando apenas actividades de lectura y escritura en sedestación. El ACT se calculó mediante el principio de dilución (ver ecuación 1) y se utilizaron para esto las concentraciones del isótopo estable en las muestras de orina basales (primera orina de la mañana) y en las muestras enriquecidas (tras 4 h de estabilización).

$\mathrm{ACT}=(\mathrm{W} . \mathrm{A} / \mathrm{a}) \times(\mathrm{Sa}-\mathrm{St}) \times \mathrm{f} /(\mathrm{Ss}-\mathrm{Sp})$

A: dosis de deuterio ingerida; a: masa de dosis de deuterio utilizada para preparar la dosis diluida; f: factor de fraccionamiento para la muestra fisiológica relativa la ACT; Sa: valor medido para la dosis diluida; Sp: valor para la muestra fisiológica predosis; Ss: valor para la muestra fisiológica; St: valor del agua de grifo utilizado en la dilución; W: masa de agua para disolver la dosis.

Las concentraciones de isótopos de hidrógeno se midieron en un espectrómetro de masas para razones isotópicas estables (PDZ Europe, Reino Unido), y se utilizaron $5 \mu$ de volumen de orina. El valor de ACT se corrigió por el intercambio isotópico (4\%) en otros compartimentos no acuosos ${ }^{15}$.

\section{Definición de sobrepeso según el porcentaje de masa grasa}

Para el diagnóstico de sobrepeso se utilizaron los valores de porcentaje de grasa publicados por Taylor et $\mathrm{al}^{16}$. Existe para cada edad un porcentaje de grasa asociado al punto de corte de $25 \mathrm{~kg} / \mathrm{m}^{2}$ de IMC, correspondiente al estándar internacional para la definición de sobrepeso ${ }^{17}$. Los valores de grasa corporal para los niños, correspondientes a un IMC de $25 \mathrm{~kg} / \mathrm{m}^{2}$, estuvieron comprendidos entre el 20-23\%, mientras que para las niñas estos valores se hallaron entre el $32-34 \%$.

\section{Análisis estadístico}

Todos los parámetros se presentaron con su valor medio y su desviación estándar (DE), así como otras medidas descriptivas. Se analizó la normalidad de la muestra mediante el test de normalidad de Kolmogorov-Smirnov. El análisis de las diferencias entre grupos de niños y niñas e individuos con y sin exceso de peso se realizó utilizando el test $\mathrm{T}$ de Student. El tamaño de la muestra se estimó utilizando el análisis de potencia estadística; se asumió un error tipo I inferior a 0,05 para las diferencias entre las variables que estimaban la adiposidad y los valores medidos. De esta forma, una muestra de 150 sujetos aseguró una potencia superior al $80 \%$ en los test estadísticos utilizados ${ }^{18}$.

El análisis mediante curvas ROC (receiver operating characteristics) permite derivar un valor de corte para la evaluación de la precisión diagnóstica de una variable que discrimine entre la ausencia y la presencia de un estado de salud (sobrepeso o normopeso $)^{19}$. En el contexto del presente estudio, el diagnóstico de precisión se refirió a la capacidad del IMC, el IMG y el IMLG como variables discriminadoras del normopeso y el sobrepeso, estimadas mediante técnicas de hidrometría y la aplicación de ecuaciones específicas de sexo y edad.

Con el uso de los puntos de corte se puede clasificar correctamente a los niños y a las niñas con sobrepeso. La sensibilidad de los índices antropométricos es la probabilidad de que éstos clasifiquen a los sujetos con sobrepeso cuando tienen verdadero sobrepeso. La especificidad es la probabilidad de que clasifiquen a los sujetos sin sobrepeso cuando verdaderamente no tienen sobrepeso (verdaderos negativos). En el análisis de curvas ROC, los verdaderos positivos (sensibilidad) se representan gráficamente contra los falsos positivos (1-especificidad) a través de todos los valores de estudio. La curva ROC estima el punto de corte, que corresponde al mejor valor con una mayor sensibilidad (1-especificidad), y muestra la mejor precisión de las variables analizadas (índices) para discriminar entre normopeso y sobrepeso. Los valores de sensibilidad y especificidad se calcularon utilizando las ecuaciones de Himes y Bouchard ${ }^{10}$. Un índice que refleja la precisión total del test es la información que da el área bajo la curva $(A B C)$. Los valores van desde $0,5-1$, y expresan la mínima o la máxima precisión del test. Los valores positivos likelihood ratio (+LR) se refieren a la existencia de un resultado positivo en presencia de la enfermedad y la posibilidad de un resultado positivo en ausencia de la enfermedad. Un valor negativo likelihood ratio (-LR) es la relación entre un resultado 
negativo en presencia de la enfermedad y la posibilidad de un resultado negativo en ausencia de la enfermedad. Los valores predictivos positivos (VPP) representan la probabilidad de que la enfermedad esté presente cuando el test es positivo, mientras que el valor predictivo negativo (VPN) es la probabilidad de no tener la enfermedad cuando el test es negativo. Se confeccionaron tablas de sensibilidad y especificidad con obtención de los puntos de corte del IMC, el IMLG y el IMG, tanto para los niños como para las niñas, con sus valores del 95\% del intervalo de confianza, para luego representar las curvas ROC. Para la realización de todos los análisis se utilizaron los programas estadísticos MedCalc (Mariakerke, Bélgica) y SPSS versión 14 (Inc, Chicago, EE. UU.) y se tomó un nivel de significación de $\mathrm{p}<0,05$.

\section{Resultados}

Las características de los sujetos estudiados se muestran en la tabla 1 . Se encontraron diferencias estadísticamente significativas

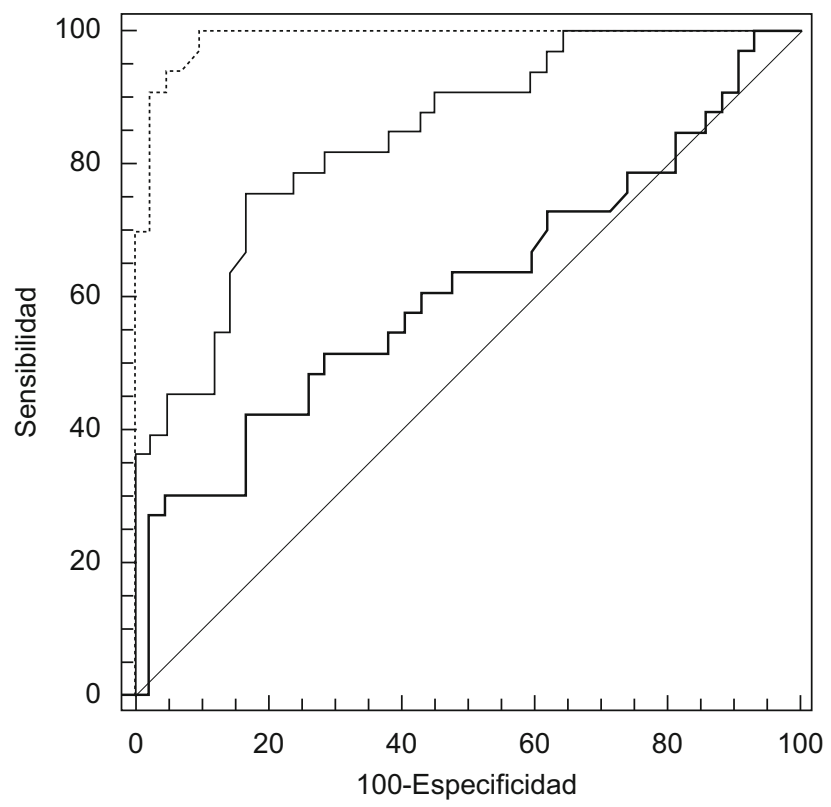

Figura 1. Curvas receiver operating characteristics de los índices de masa grasa (---), índice de masa corporal (---) e índice de masa libre de grasa (-) en los niños.

Tabla 2

Áreas bajo la curva receiver operating characteristics, error estándar e intervalos de confianza del $95 \%$ en los niños

\begin{tabular}{llll}
\hline Variable & ABC & EE & IC del 95\% \\
\hline IMG & 0,988 & 0,014 & $0,930-0,998$ \\
IMLG & 0,612 & 0,066 & $0,492-0,722$ \\
IMC & 0,842 & 0,048 & $0,740-0,916$ \\
\hline
\end{tabular}

ABC: área bajo la curva; EE: error estándar; IC: intervalo de confianza; IMC: índice de masa corporal; IMG: índice de masa grasa; IMLG: índice de masa libre de grasa. en el peso y la talla, con valores mayores en los niños. Los sumatorios de pliegues cutáneos de 6 y 8 pliegues fueron mayores en las niñas $(p<0,01$ y $p=0,045)$. Los valores de masa grasa no difirieron entre los grupos, pero sí fue mayor la MLG, el IMC y el IMLG en los niños ( $\mathrm{p}<0,05$ ). El \%MG y el IMG fueron mayores en las niñas que en los niños $(\mathrm{p}<0,001)$.

\section{Curvas receiver operating characteristics en los niños}

Los análisis de las curvas ROC para los niños se muestran en la figura 1 y en las tablas 2 y 3. Los valores de la curva ROC y su índice $A B C$ tuvieron un valor muy alto para el IMG $(A B C=0,988)$ y un valor alto para el IMC ( $A B C=0,84)$. El $A B C$ para el IMLG no fue significativa ni capaz de predecir el sobrepeso en los niños ( $A B C=0,612)$. Como resultado, podemos observar diferencias muy significativas entre las $A B C$ del IMC y del IMG con respecto al IMLG $(\mathrm{p}<0,001)$.

El punto de corte de $4,58 \mathrm{~kg} / \mathrm{m}^{2}$ del IMG resultó poseer un $100 \%$ de sensibilidad y un $90,5 \%$ de especificidad, y el punto de corte del IMC fue de $23,07 \mathrm{~kg} / \mathrm{m}^{2}$, con unos valores del $75,8 \%$ y del $83,3 \%$ de sensibilidad y especificidad, respectivamente. Por el contrario, el IMLG presentó unos índices de sensibilidad del 42,4\% y de especificidad del $83,3 \%$, y resultó tener una deficiente capacidad para detectar el sobrepeso. El IMG tuvo un +LR de 10,5 y un -LR de 0 , lo que le otorga una capacidad diagnóstica muy grande sobre el IMC y más sobre el IMLG. Asimismo, los VPP y los VPN fueron mucho mayores para el IMG y el IMC (tabla 3).

\section{Curvas receiver operating characteristics en las niñas}

Las curvas ROC para las niñas se muestran en la figura 2 y en las tablas 4 y 5 . Los valores de la curva $R O C$ y su índice $A B C$ tuvieron valores muy altos para el IMG $(A B C=0,996)$ y un valor moderadamente alto para el IMC $(A B C=0,828)$. El $A B C$ para el IMLG tampoco fue significativa ni capaz de predecir el sobrepeso en las niñas $(A B C=0,508)$. Existieron también diferencias muy significativas entre las $A B C$ del IMG y del IMC con respecto al IMLG en las niñas ( $p<0,001)$.

El punto de corte de $7,76 \mathrm{~kg} / \mathrm{m}^{2}$ del IMG resultó poseer un $100 \%$ de sensibilidad y un $97 \%$ de especificidad, y el punto de corte del IMC fue de $23,18 \mathrm{~kg} / \mathrm{m}^{2}$, con valores del 88,9 y del $77,3 \%$ de sensibilidad y especificidad, respectivamente. Por el contrario, el IMLG tuvo unos índices de sensibilidad del 45,2\% y de especificidad del 73,8\%, y resultó tener una deficiente capacidad para detectar el sobrepeso. El IMG tuvo un +LR de 33,0 y un -LR de 0, lo que le otorgó una capacidad diagnóstica muy grande sobre el IMC y el IMLG. Asimismo, los VPP y los VPN fueron mucho mayores para el IMG.

\section{Discusión}

Los resultados del presente estudio muestran que el IMG puede utilizarse con razonable éxito y precisión para detectar el sobrepeso en jóvenes adolescentes de ambos sexos con una edad

Tabla 3

Sensibilidad y especificidad de los valores de corte del índice de masa grasa, el índice de masa libre de grasa y el índice de masa corporal en los niños

\begin{tabular}{|c|c|c|c|c|c|c|c|}
\hline & Punto de corte & Sensibilidad (IC del 95\%) & Especificidad (IC del 95\%) & $+\mathrm{LR}$ & $-\mathbf{L R}$ & VPP & VPN \\
\hline IMG & 4,58 & $100(89,3-100,0)$ & $90,5(77,4-97,3)$ & 10,50 & 0,00 & 89,2 & 100,0 \\
\hline IMLG & 19,15 & $42,4(25,5-60,8)$ & $83,3(68,6-93,0)$ & 2,55 & 0,69 & 66,7 & 64,8 \\
\hline IMC & 23,07 & $75,8(57,7-88,9)$ & $83,3(68,6-93,0)$ & 4,55 & 0,29 & 78,1 & 81,4 \\
\hline
\end{tabular}

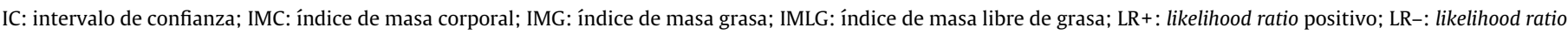
negativo; VPN: valor predictivo negativo; VPP: valor predictivo positivo. 
en el intervalo de entre los 12 - 18 años. Así, los puntos de corte del IMG otorgan la mejor relación entre la sensibilidad y la especificidad y se muestran como parámetros válidos para detectar y discriminar entre el normopeso y el sobrepeso. La utilización del IMC y del IMG como sistema de valoración de la adiposidad corporal total resultan de gran importancia para la evaluación clínica y habitual de la composición corporal, pues es una herramienta simple que puede ayudar en la selección de diagnósticos preliminares de ciertas enfermedades, como la diabetes de tipo II o las enfermedades del ámbito cardiovascular. Gran número de estudios demuestran la validez del uso del IMC $^{3,6,20-26}$ y del IMG $^{9}$ como índices válidos para discriminar el sobrepeso en población adulta; sin embargo, estos puntos de corte no se ajustan bien en poblaciones adolescentes. Nuestros resultados muestran nuevos valores de corte para el IMC y el IMG como predictores del sobrepeso, lo que ofrece una herramienta relativamente simple para el control del sobrepeso en la

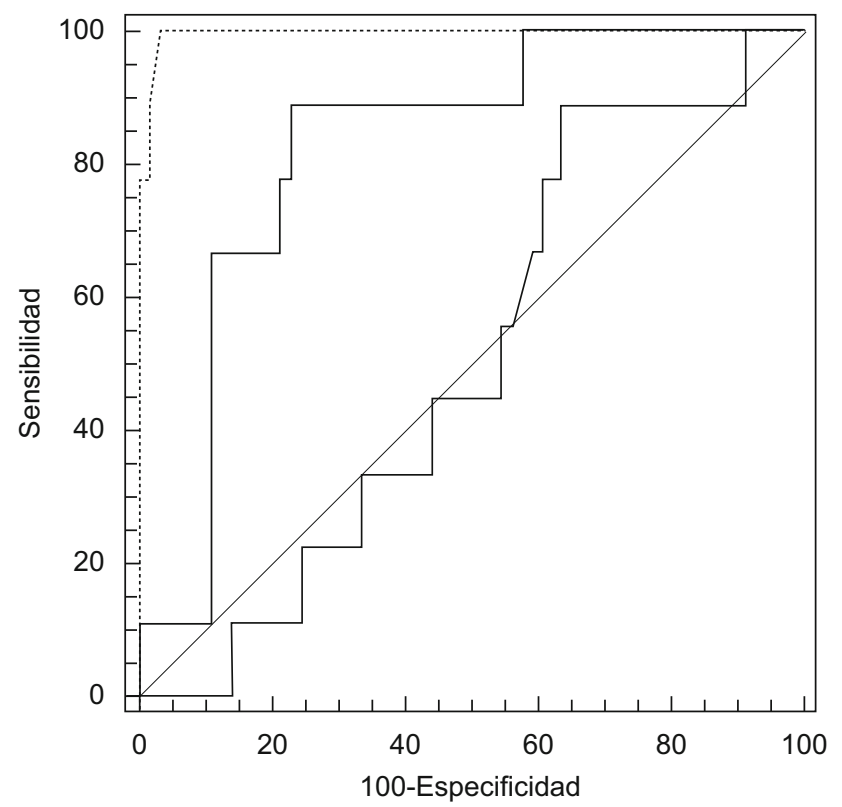

Figura 2. Curvas receiver operating characteristics de los índices de masa grasa (-), índice de masa corporal (-) e índice de masa libre de grasa (-) en las niñas.

Tabla 4

Áreas bajo la curva receiver operating characteristics, error estándar e intervalo de confianza del $95 \%$ en las niñas

\begin{tabular}{llll}
\hline Variable & ABC & EE & IC del 95\% \\
\hline IMG & 0,996 & 0,015 & $0,943-1,000$ \\
IMLG & 0,508 & 0,104 & $0,390-0,626$ \\
IMC & 0,828 & 0,087 & $0,724-0,906$ \\
\hline
\end{tabular}

ABC: área bajo la curva; EE: error estándar; IC: intervalo de confianza; IMC: índice de masa corporal; IMG: índice de masa grasa; IMLG: índice de masa libre de grasa. población infantil mediante el uso de índices antropométricos de fácil obtención. Los resultados derivados de nuestra muestra de población malagueña estuvieron próximos a otros derivados en poblaciones de la Península Ibérica. Así, un estudio realizado en escolares portugueses ${ }^{27}$ sobre la sensibilidad y la especificidad de algunas medidas antropométricas mostró que el pliegue tricipital posee una alta sensibilidad y una baja especificidad para el diagnóstico de la obesidad. Otras variables analizadas, como el IMC y el perímetro del brazo, poseen valores de sensibilidad y especificidad que permiten su aplicación razonablemente como herramienta diagnóstica de la obesidad. Los puntos del IMC recomendados para la población de jóvenes portugueses $^{27}$ son parecidos a los de nuestro estudio (puntos de corte de $19-24 \mathrm{~kg} / \mathrm{m}^{2}$ para los niños y de $21-22 \mathrm{~kg} / \mathrm{m}^{2}$ para las niñas). Por otro lado, los valores medios de peso, talla e IMC del grupo de estudio son semejantes a los aportados en otros estudios con escolares adolescentes españoles ${ }^{28}$. De este modo, nuestros valores de corte deberían ofrecer un buen grado de generabilidad y aplicabilidad ya que el uso de la antropometría es de fácil aplicación en numerosos contextos clínicos y que las características físicas de nuestra muestra no fueron diferentes de otras dentro del mismo escalón etario. Sin embargo, la derivación de valores de corte a través del análisis de curvas ROC no siempre se ha utilizado, y los valores de corte utilizados como diagnóstico para el sobrepeso y la obesidad por parte de comités de expertos ${ }^{30}$ recomiendan el control preventivo del sobrepeso y la obesidad cuando en los jóvenes el IMC es mayor o igual al percentil 95 para cada edad y sexo o mayor a $30 \mathrm{~kg} / \mathrm{m}^{2}$ (obesidad), o cuando en los adolescentes los valores están entre el percentil > 85 y 95 o bien son menores a $30 \mathrm{~kg} / \mathrm{m}^{2}$ (exceso de peso). De acuerdo con los valores de IMC descritos por Himes y Dietz ${ }^{30}$ para los niños con edades comprendidas entre 12 - 18 años, el riesgo de sobrepeso se instala cuando están entre $25-30 \mathrm{~kg} / \mathrm{m}^{2}$, mientras que para las niñas este riesgo se sitúa para valores entre $26-30 \mathrm{~kg} / \mathrm{m}^{2}$, lo que debe representar el mismo valor que para los adultos. Los valores del presente estudio se hallan por debajo de éstos y corresponden a $23,07-23,18 \mathrm{~kg} / \mathrm{m}^{2}$ para los niños y las niñas, respectivamente; estos valores son mucho más representativos de la biología madurativa de los adolescentes ya que las curvas de peso y talla no tienen una evolución homogénea dentro del ciclo vital de los jóvenes ${ }^{13}$. De esta forma, el incremento de la adiposidad asociado al incremento de peso no debe guardar la misma razón de proporcionalidad que en los adultos. Un ejemplo de esta situación se da entre los adolescentes de sexo masculino, en los que el incremento de la producción de testosterona a partir de la pubertad promueve un incremento de la masa muscular esquelética y de la estatura, lo que, sin duda, altera la razón de proporcionalidad entre el incremento de estatura y la masa corporal (grasa y magra).

Un estudio de Mei et $\mathrm{al}^{22}$ analiza en niños y adolescentes de 2 - 19 años la sensibilidad y la especificad de índices como el IMC, el índice de Rohrer y el índice de peso/talla. Al analizar el grupo de 12 - 19 años, similar en edad al de nuestro estudio, encuentran que el IMC presenta un $A B C$ media de 0,95 (DE de 0,006), muy similar al nuestro aunque ligeramente inferior. Los valores de

Tabla 5

Sensibilidad y especificidad de los valores de corte del índice de masa grasa, el índice de masa libre de grasa y el índice de masa corporal en las niñas

\begin{tabular}{|c|c|c|c|c|c|c|c|}
\hline & Punto de corte & Sensibilidad (IC del 95\%) & Especificidad (IC del 95\%) & $+\mathrm{LR}$ & $-\mathbf{L R}$ & VPP & VPN \\
\hline IMG & 7,76 & $100(66,2-100)$ & $97,0(89,5-99,5)$ & 33,00 & 0,00 & 81,8 & 100 \\
\hline IMLG & 14,64 & $88,9(51,7-98,2)$ & $36,4(24,9-49,1)$ & 1,40 & 0,31 & 16,0 & 96,0 \\
\hline IMC & 23,18 & $88,9(51,7-98,2)$ & $77,3(65,3-86,7)$ & 3,91 & 0,14 & 34,8 & 98,1 \\
\hline
\end{tabular}

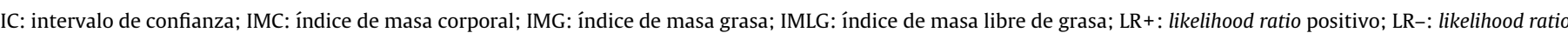
negativo; VPN: valor predictivo negativo; VPP: valor predictivo positivo. 
sensibilidad y especificidad para el IMC, a partir de los datos conjuntos de la muestra de Mei et $\mathrm{al}^{22}$, son del $100 \mathrm{y}$ del $72 \%$, respectivamente (prácticamente iguales a los encontrados en nuestro estudio), por lo que pueden predecir con alta seguridad tanto los valores verdaderos negativos como los valores falsos positivos. En los niños, el IMG presenta valores de sensibilidad máximos (100\%), por lo que el test obtiene una valoración correcta en el $100 \%$ de los casos en los que el niño presenta sobrepeso; la especificidad de este índice es también es muy alta (90,5\%). En el caso del IMC, los valores de sensibilidad $(75,8 \%)$ y especificidad $(83,3 \%)$ se consideran moderadamente altos en comparación con el IMG. Estos resultados están en desacuerdo con los aportados por Himes y Bouchard ${ }^{10}$ y Malina y Katzmarzyk ${ }^{20}$, que manifiestan que el IMC, el peso y algunos pliegues de grasa (tríceps y subescapular) tienen una gran especificidad y una baja sensibilidad. Por esta razón, la utilización de los valores propuestos por estos últimos autores podría llevarnos con facilidad a cometer un error tipo II (falso negativo). Dicho de otra forma, el valor diagnóstico de sus valores de corte tiene una baja probabilidad de clasificar correctamente a los sujetos con sobrepeso. Por el contrario, al igual que sucede con nuestros valores, sí tienen una buena capacidad diagnóstica para clasificar a sujetos con normopeso.

En el grupo de niñas, el IMG presenta valores de sensibilidad máximos (100\%) y se obtiene una valoración correcta en el $100 \%$ de los casos en que la niña tiene sobrepeso. La especificidad del IMG es también muy alta (97\%). En el caso del IMC, los valores de sensibilidad y especificidad se consideran también moderadamente altos: corresponden a unos valores del 88,9 y del $77,3 \%$, respectivamente. En este caso del grupo de niñas, también los valores de sensibilidad y especificidad estarían en concordancia con los trabajos de Himes y Bouchard ${ }^{10}$. Estas diferencias entre los valores del estudio precedente y los del presente estudio pueden deberse a la mayor heterogeneidad de la muestra de Himes y Bouchard (entre 8-18 años). Además, en su trabajo se utilizó el método densitométrico para estimar el \%MG, lo que debió incrementar el error de los métodos debido a que la maduración bioquímica de los niños prepúberes incluidos en la muestra es diferente a la de los adolescentes ${ }^{14}$, lo que puede influir de manera definitiva en la validez del modelo de 2 componentes utilizado para derivar el \%MG, fundamentalmente por el error introducido en las constantes de densidad de la MLG ${ }^{14}$.

Los valores del ABC para el IMLG tanto en los niños como en las niñas son de 0,612 y de 0,508 , respectivamente, con un intervalo de confianza del $95 \%$ que incluye el valor de 0,5 ; por tanto, quedan invalidados como índices con una buena sensibilidad y especificidad diagnóstica. Además de los índices propuestos en este estudio, existen también otros índices o variables antropomé$\operatorname{tricas}^{24,31}$, como son los pliegues de grasa, que también se han recomendado extensamente por su relación con la grasa corporal total y han servido para derivar ecuaciones de cálculo de la grasa corporal total. Sin embargo, la existencia de correlaciones entre la grasa corporal y los pliegues de grasa no describen completamente la naturaleza de la clasificación de las personas en sujetos con normopeso o sujetos con sobrepeso. Para estos propósitos y por lo anteriormente dicho, es recomendable realizar análisis categóricos, los que requieren una medida o un índice válido para detectar el exceso de adiposidad per $\mathrm{se}^{28}$.

Debido a la ausencia de una definición ampliamente aceptada para definir puntos de corte para el exceso de adiposidad en jóvenes por sexo y edad, se han utilizado referencias de poblaciones semejantes a la nuestra ${ }^{16}$. La fuerza del estudio de Taylor et $\mathrm{al}^{16}$ reside en que se ha realizado en una gran muestra, con la utilización de un método validado para estudios de composición corporal - como es la absorciometría de rayos $\mathrm{X}$ de doble energía-, así como una validación de las medidas de grasa corporal en niños mediante modelos multicompartimentales, que permiten una mayor precisión, menores errores de estimación y su relación con los puntos de corte internacionales ${ }^{17}$. No obstante, todavía no existen valores de corte del \%MG bien definidos para detectar la obesidad patológica. Así pues, nuestros valores de corte apenas se refieren al estado de exceso ponderal que puede asociarse a determinadas comorbilidades.

Las limitaciones del presente estudio pueden estar en el tamaño de la muestra (150 sujetos); pero se recogió también el peso y la talla de una muestra total del entorno colegial $(n=450)$, y los valores medios de las variables antropométricas básicas fueron el peso $(60,85 \mathrm{~kg}$ [DE de 14,24$])$, la talla $(162,78 \mathrm{~cm}$ [DE de 8,14$])$ y el IMC $\left(22,8 \mathrm{~kg} / \mathrm{m}^{2}\right.$ [DE de 4,48]), y las medias de éstos en la población general no fueron significativamente diferentes de aquéllas de la muestra de estudiada, por lo que no debería existir sesgo relativo al muestreo. Por otro lado, como se comentó en los primeros párrafos, nuestra muestra es representativa de la población española de referencia ${ }^{28,29}$ y de otros países del sur de Europa ${ }^{27,32}$ en cuanto a las variables antropométricas para cada edad y sexo.

La aplicación de los puntos de corte del IMG en niños de $4,58 \mathrm{~kg} / \mathrm{m}^{2}$ y en niñas de $7,76 \mathrm{~kg} / \mathrm{m}^{2}$ posee una gran sensibilidad y especificidad como indicador de sobrepeso en adolescentes. No obstante, la aplicación de los puntos de corte del IMC en niños de $23,07 \mathrm{~kg} / \mathrm{m}^{2}$ y en niñas de $23,18 \mathrm{~kg} / \mathrm{m}^{2}$ también posee una gran sensibilidad y especificidad como indicador de sobrepeso en adolescentes, y se podría utilizar en aquellos contextos donde la aplicación de series antropométricas es inviable o donde no existen recursos técnicos o humanos para su realización. Por esto, la facilidad de la toma de estas variables antropométricas básicas, con bajo error y buena reproducibilidad ofrece la posibilidad a través de índices simples (IMG o, en su defecto, IMC) de la valoración de la adiposidad corporal total desde un punto de vista clínico, lo que constituye una herramienta de valiosa importancia para la clasificación diagnóstica del sobrepeso de los adolescentes españoles.

\section{Conflicto de intereses}

Los autores declaran no tener ningún conflicto de intereses.

\section{Agradecimientos}

Expresamos nuestro mayor agradecimiento a los participantes en el estudio, a la Dirección y a los profesores del IES Nuestra Sra. de la Victoria de Málaga por su cooperación e interés, y al apoyo de la Consejería de Educación de la Junta de Andalucía.

\section{Bibliografía}

1. World Health Organization. Obesity: Preventing and managing the global epidemic. Report of a WHO consultation on obesity. Geneva: WHO; 1998

2. González E, Palmeros C, Villanueva J, Torres B, Bastida S, Vaquero MP, et al. Prevalencia del síndrome metabólico y su asociación con el índice de masa corporal en universitarios. Med Clin (Barc). 2007;129:766-9.

3. Toledo E, Beunza JJ, Núñez-Córdoba JM, Bes-Rastrollo M, Basterra FJ, MartínezGonzález MA. Metabolic risk factors in a cohort of young adults and their association with a body-mass index between 22 and $25 \mathrm{~kg} / \mathrm{m}^{2}$. Med Clin (Barc). 2009;132:654-60.

4. Roubenoff R, Dallal GE, Wilson PWF. Predicting body fatness: The body mass index vs. estimation by bioelectrical impedance. Am J Public Health. 1995;85:726-8.

5. Baumgartner RN, Heymsfield SB, Roche AV. Human body composition and the epidemiology of chronic disease. Obes Res. 1995;3:73-95.

6. Troiano RP, Flegal KM, Kuczmarski RJ, Campbell SM, Johnson CL. Overweight prevalence and trends for children and adolescents: The National Health and Nutrition Examination Surveys, 1963 to 1991. Arc Pediatr Adolesc Med. 1995;149:1085-91. 
7. Dietz WH, Robinson TN. Use of the body mass index (BMI) as a measure of overweight in children and adolescents. J Pediatr. 1998;132:191-3.

8. Alvero Cruz JR, Diego Acosta AM, Fernández Pastor VJ, García Romero J. Métodos de evaluación de la composición corporal: tendencias actuales I. Archivos Medicina del Deporte. 2004;104:535-40.

9. Van Itallie TB, Yang MU, Heymsfield SB, Funk RC, Boileau RA. Height normalized indices of the body's fat-free mass and fat mass: Potentially useful indicators of nutritional status. Am J Clin Nutr. 1990;52:953-9.

10. Himes JH, Bouchard C. Validity of anthropometry in classifying youths as obese. Int J Obes. 1989;13:183-93.

11. Alvero JR, Carnero EA, Barrera J, Fernández JC, Carrillo M, Martin MC, et al. The Esccola Study: health, nutrition, body composition and physical fitness in Spanish adolescents. Abstract Book. Helena Symposium: Promoting Healthy European Lifestyle through Exercise and Nutrition in Adolescence. Universidad de Granada (España). 2008, p. 54.

12. Norton K, Whittingham N, Carter L, Kerr D, Gore C. Measurement techniques in anthropometry. En: Norton K, Olds T, editores. Anthopometrica. Sydney: University of New South Wales Press; p. 75-25.

13. Fomon SJ, Haschke F, Ziegler EE, Nelson SE. Body composition of reference children from birth to age 10 years. Am J Clin Nutr. 1982;35:1169-75.

14. Lohman TG. Applicability of body composition techniques and constants of children and youths. Exer Sports Sci Rev. 1986;14:325-57.

15. Schoeller DA. Hydrometry. En: Heymsfield SB, Lohman T, Wang Z, Going SB, editores. Human Body Composition. Champaign, IL: Human Kinetics; 1996. p. 35-49.

16. Taylor RW, Jones IE, Williams SM, Goulding A. Body fat percentages measured by X-ray absortiometry corresponding to recently recommended body mass index cutoffs for overweight and obesity in children and adolescents aged 3-18 y. Am J Clin Nutr. 2002;76:1416-21.

17. Cole TJ, Bellizzi MC, Flegal KM, Dietz VH. Establishing a standard definition for child overweight and obesity worldwide: International survey. BMJ. 2000;320:1-6.

18. Cohen J. Statistical power analysis for the behavioral sciences, 2nd ed. Lawrence Erlbaum Assoc Hillsdale, NJ; 1988.

19. Zweig MH, Campbell G. Receiver -operating characteristics (ROC) plots: A fundamental evaluation tool in clinical medicine. Clin Chem. 1993;39:561-77.
20. Malina RM, Katzmarzyk PT. Validity of the body mass index as an indicator of the risk and presence of overweight in adolescents. Am J Clin Nutr. 1999;70:131S-6S.

21. Janssen I, Katzmarzyk PT, Ross R, Leon AS, Skinner JS, Rao DC, et al. Fitness alters the association of BMI and waist circumference with total and abdominal fat. Obes Res. 2004;12:525-37.

22. Mei Z, Grummer-Strawn LM, Pietrobelli A, Goulding A, Goran MI, Diets WH. Validity of body mass index compared with other body-composition screening indexes for the assessment of body fatness in children and adolescents. Am J Clin Nutr. 2002;75:978-85.

23. Rossner B, Prineas N, Loggie J, Daniels SR. Percentiles for body mass index in US children 5 to 17 years of age. J Pediatr. 1998;132:211-22.

24. Roche AF, Sievogel RM, Chumlea WC, Webb P. Grading body fatness from limited anthropometric data. Am J Clin Nutr. 1981;34:2831-8.

25. Eto C, Komiya S, Nakao T, Kikkawa K. Validity of the body mass index as an indicator of obesity in children aged 3.5 year. J Physiol Anthropol Appl Human Sci. 2004;23:25-30.

26. Pietrobelli A, Faith MS. Body mass index is a measure of adiposity among children and adolescents: A validation study. J Pediatr. 1998;132:204-10.

27. Sardinha LB, Going SB, Teixeira PJ, Lohman TG. Receiver operating characteristics analysis of body mass index, triceps skinfold and arm girth for obesity screening in children and adolescents. Am J Clin Nutr. 1999;70:1090-5.

28. Moreno LA, Mesana MI, González-Gross M, Gil CM, Wärnberg J, Ruiz JR, et al., and the AVENA Group Study. Anthropometric body fat composition reference values in Spanish adolescents. The AVENA Study. Eur J Clin Nutr. 2005:1-6.

29. Henríquez P, Doreste J, Laínez P, Estevez MD, Iglesias M, Martin G, et al Prevalencia de obesidad y sobrepeso em adolescentes canaries. Relación con el desayuno y la actividad física. Med Clin (Barc). 2008;130:606-10.

30. Himes JH, Dietz WH. Guidelines for overweight in adolescent preventive services: Recommendations for an expert committee. Am J Clin Nutr. 1994;59:307-16.

31. Lohman TG. Skinfold and body density and their relation to body fatness: A review. Hum Biol. 1981;53:181-225.

32. Do Carmo I, Dos Santos O, Camolas J, Vieira J, Carreira M, Medina L, et al. Overweight and obesity in Portugal: National prevalence in 2003-2005. Obes Rev. 2008;9:11-9. 